\title{
Cooperative Wireless Multicell OFDMA Network with Backhaul Capacity Constraints
}

\author{
Aakanksha Chowdhery*, Wei Yu ${ }^{\dagger}$, John M. Cioffi* \\ ${ }^{*}$ Stanford University, Stanford, CA, USA $\quad{ }^{\dagger}$ University of Toronto, Ontario, Canada \\ achowdhe@stanford.edu,weiyu@comm.utoronto.ca, cioffi@stanford.edu
}

\begin{abstract}
This paper considers the downlink of a wireless multicell orthogonal frequency division multiple-access (OFDMA) system where neighboring base-stations (BSs) can jointly encode and transmit data signals to their users using zero-forcing (ZF) precoding in each frequency tone, but only limited backhaul capacity is available for each BS to share its users' data streams with the neighboring BSs. A numerical algorithm is proposed to maximize the network-wide utility of this system subject to backhaul-capacity constraints. The proposed algorithm first selects a subset of frequency tones for each BS to share its users' data streams with the neighboring BSs and then, jointly schedules users and adapts the precoding coefficients and the power spectra of the BSs to effectively utilize the limited backhaul capacity. Numerical results show that using the proposed algorithm, the gain in downlink sum-rate per cell can be made to scale linearly with the available backhaul capacity per BS between the no-cooperation and the full-cooperation scenarios.
\end{abstract}

\section{INTRODUCTION}

Multicell cooperative processing promises to significantly improve the performance of wireless cellular networks by coordinating multiple base-stations (BSs) for the effective mitigation of intercell interference [1]-[4]. The realization of this promise, however, comes at the cost of very highcapacity backhaul communication between the cooperating base-stations. This is because as multiple BSs jointly encode and transmit data signals to their users in multicell cooperative processing, the data streams of their users must be shared among all the cooperating BSs. The cost and implementation complexity of such high-capacity backhaul communication can be prohibitive in a practical implementation. This paper aims to answer the following question: How much of the performance gain of multicell cooperative processing can still be retained in a wireless multicell system if only limited backhaul capacity is available to each BS to share its users' data streams with the neighboring BSs?

This paper considers the downlink of a wireless multicell orthogonal frequency division multiple-access (OFDMA) network where a BS can use zero-forcing (ZF) precoding to pre-subtract the intercell interference of the cooperating neighboring BSs. Cooperation between any two neighboring BSs requires sharing of the user data-streams over backhaul. Therefore, in order to reduce the total volume of backhaul communication, this paper proposes that each BS chooses a subset of frequency tones for each of its neighboring BSs, and shares the users' data only on this subset of tones. Thus, on a particular frequency tone, each BS may share its users' data with only a subset of the neighboring BSs as in the example in Fig. 1.

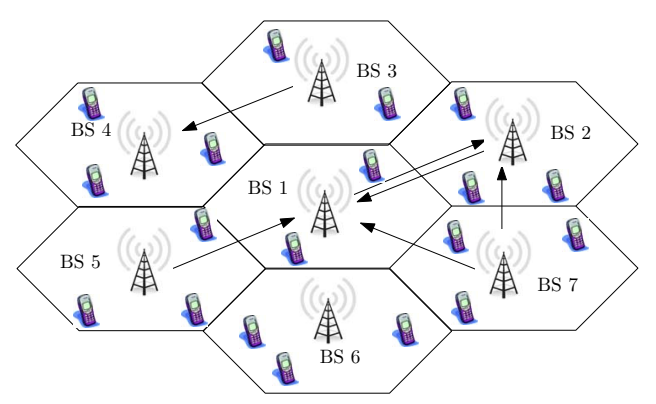

Figure 1. Wireless Multicell Scenario with each BS cooperating with only a subset of the neighboring BSs in the $n$th frequency tone. The direction of arrow on the cooperation link indicates that the source BS sends its user data to the destination BS and then, the destination BS pre-subtracts the intercell interference of the source BS using $\mathrm{ZF}$ precoding.

For this strategy to be effective, one must be able to efficiently solve the network optimization problem associated with the proposed scheme. Toward this end, this paper adopts a network optimization framework of maximizing a networkwide utility function while satisfying the backhaul-capacity constraints of each BS. To achieve a network-wide optimal solution under the utility maximization objective, all BSs must jointly optimize the following on all frequency tones: the user schedules, the precoder coefficients, the transmit power spectra, and the subset of frequency tones on which they share their users' data streams with each of the neighboring BSs for ZF precoding. Clearly, finding a network-wide global optimal solution for such a complex network optimization problem is likely to be impractical. This paper therefore focuses on a heuristic approach that first judiciously chooses the cooperation links (for sharing the user's data streams) for each frequency tone at each BS, while accounting for both the benefit and the cost of selecting the links. The proposed algorithm then jointly schedules users and adapts the zeroforcing precoder coefficients as well as the power spectra of all BSs using an iterative approach.

We show in this paper that the above numerical approach is in fact feasible in a practical network. Further, we provide numerical evidence to suggest that the benefit of multicell cooperative processing (in term of downlink sum-rate improvement per cell) in fact scales linearly with the backhaulcapacity constraint per BS between the no-cooperation and the full-cooperation scenarios. Note that the full-cooperation scenario requires a backhaul-capacity of six times the downlink sum-rate per cell (for a hexagonally tiled cellular network in which each BS has to share its users' data streams with each of the six neighboring BSs). Using the proposed algorithm, 
this paper shows that if only $50 \%$ of the full-cooperation backhaul capacity is available per BS, then $50 \%$ of the fullcooperation gain in downlink sum-rate can still be retained as compared to the no-cooperation scenario. The proportional fairness objective of the proposed algorithm ensures that the gain in data-rates is fairly distributed among all the users.

\section{A. Related Work}

To reduce backhaul communication in the single-frequency wireless multicell networks, the approach of clustering the BSs into disjoint clusters has been proposed in [5]-[9]. The cooperation cluster comprises a limited number of BSs, thus backhaul communication is required only within the cluster. However, although intra-cluster intercell interference is eliminated completely, each cluster still receives interference from other clusters. The BSs in a cluster can be chosen statically [7], [8] or dynamically in time [5]. The concept of clustering may be applied independently to each frequency tone in an OFDMA network to reduce backhaul communication. However, ensuring backhaul-capacity constraints over all frequency tones requires a combinatorial search for cluster-selection in each of the frequency tones, which is not a trivial task. To this end, a different approach is considered for backhaulconstrained wireless multicell OFDMA networks in [9] where cooperation links are added only for the weakest set of users in the no-cooperation scenario until each of the finite-capacity backhaul links between BSs are used up.

The proposed multicell model with limited backhaul communication is related to the information theoretical study of interference channel with rate-limited cooperation at the transmitter. In this realm, a shared-private rate-splitting scheme is proposed in [10], where each transmitter shares only a part of its message with the other transmitter for partial zeroforcing precoding, and where the private message remains as interference. This paper considers a considerably simpler approach where the transmitter shares either no message or the entire message in each frequency tone, but messages are shared on only a subset of frequency tones for ZF precoding.

\section{SySTEM MOdEL}

Consider an interference-limited network with $L$ cells, $K$ users per cell and an OFDMA multiplexing scheme with $N$ tones over a fixed bandwidth. The frequency assignments for users within each cell are non-overlapping. Thus, users experience only intercell interference and no intracell interference. Both the base-station and the mobile-user are equipped with a single antenna. Multipath fading channels are initially estimated between all the BSs and the remote terminals in the same cell as well as the neighboring cells on all frequency tones, and this channel-state information is made available to every BS. The channel-state information (CSI) is assumed to be perfect in this paper.

Each base-station is connected to its neighboring BSs via backhaul links. Further, limited backhaul capacity is assumed to be available to each base-station to share its users' data streams with the neighboring BSs for ZF precoding. The backhaul capacity of a base-station may not be sufficient for sharing its users' data streams with all the neighboring BSs in all the frequency tones and thus, the base-station needs to choose a subset of frequency tones to share its users' data streams with the neighboring BSs. In this paper, additional overhead incurred by transmitting the channel-state information over the backhaul is not considered, though the reader may find an interesting study of the same in [11].

Each base-station computes the ZF precoding coefficients to pre-subtract the intercell interference of its cooperating neighboring BSs. The cooperation links are directional. A cooperation link from the $l$ th base-station to the $m$ th basestation in the $n$th tone means that the $l$ th base-station sends the data stream of its scheduled user in the $n$th tone to the $m$ th base-station via backhaul. So, the $m$ th base-station uses zeroforcing precoding to pre-subtract intercell interference from the $l$ th cell to the scheduled user in its own cell in the $n$th tone. Therefore, each outgoing cooperation link can reduce the interference to and increase the data rate of the destination BS, but it also incurs an additional cost of transmitting the user's data stream over the backhaul.

Let $p_{m}^{n}$ represent the power spectrum used by the $m$ th basestation to transmit signal to the scheduled user in its own cell in the $n$th tone and $u_{m k}^{n} \in \mathbb{C}$ represent the information signal for the $k$ th user in the $m$ th cell in the $n$th tone, which is assumed to have unit energy. The downlink user schedules are determined by the assignment function $f(m, n)$, which assigns a user $k$ to the $m$ th cell in the $n$th tone for downlink, i.e. $k=f(m, n)$. Since the $m$ th BS uses its transmit power to transmit data to its scheduled user and to zero-force the signal of scheduled users in other cells which cooperate with the $m$ th cell, the transmitted signal $x_{m}^{n}$ of the $m$ th base-station in the $n$th tone is of the form

$$
x_{m}^{n}=\sqrt{p_{m}^{n}} u_{m k}^{n}+\sum_{l=1, l \neq m}^{L} w_{m k, l k^{\prime}}^{n} \sqrt{p_{l}^{n}} u_{l k^{\prime}}^{n},
$$

where $w_{m k, m k}^{n}=1$ and $\left\{w_{m k, l k^{\prime}}^{n} \forall m \neq l\right\}$ represents the precoding coefficient used by the $m$ th BS to pre-subtract the intercell interference caused by the $l$ th cell to its own scheduled user $k$ in the $n$th tone. Here $k^{\prime}$ is the user scheduled at the $l$ th base-station, i.e. $k^{\prime}=f(l, n)$. Note that $w_{m k, l k^{\prime}}^{n}=0$ if there is no cooperation link from the $l$ th base-station to the $m$ th base-station in the $n$th tone because the $m$ th base-station does not receive the $l$ th base-station's user data.

Let $h_{l, m k}^{n}$ denote the complex channel response between the $l$ th base-station and the $k$ th remote user in the $m$ th cell in the $n$th tone for downlink. Let $\mathbf{h}_{m}^{n}$ be a $L \times 1$ column vector obtained by stacking the channel gains $\left\{h_{l, m k}^{n} \forall l\right\}$ of all the BSs to the scheduled user $k$ in the $m$ th cell. Then, the received signal at the $k$ th user in the $m$ th cell scheduled in the $n$th tone can be written as

$$
y_{m k}^{n}=\sum_{l=1}^{L}\left(\mathbf{h}_{m}^{n}\right)^{H} \mathbf{w}_{l}^{n} \sqrt{p_{l}^{n}} u_{l k^{\prime}}^{n}+z_{m k}^{n},
$$


where the precoding vector $\mathbf{w}_{m}^{n} \in \mathbb{C}^{L \times 1}$ is obtained by stacking the precoder coefficients $\left\{w_{l k^{\prime}, m k}^{n} \forall l\right\}$ used by all the BSs to zero-out the interference caused by the $m$ th cell and $w_{m k, m k}^{n}=1$. Here, $z_{m k}^{n}$ is the additive white Gaussian complex noise with variance $\sigma^{2} / 2$ on each of its real and imaginary components, $k=f(m, n), k^{\prime}=f(l, n)$ and $(.)^{H}$ is the hermitian operator.

The signal-to-interference ratio (SINR) of the $k$ th user in the $m$ th cell scheduled in the $n$th tone is given by

$$
\operatorname{SINR}_{m k}^{n}=\frac{\left|\left(\mathbf{h}_{m}^{n}\right)^{H} \mathbf{w}_{m}^{n}\right|^{2} p_{m}^{n}}{\Gamma\left(\sum_{l \neq m, l=1}^{L}\left|\left(\mathbf{h}_{m}^{n}\right)^{H} \mathbf{w}_{l}^{n}\right|{ }^{2} p_{l}^{n}+\sigma^{2}\right)},
$$

where $\Gamma$ is the SNR gap corresponding to the choice of modulation and coding schemes. The instantaneous downlink user rate for the $k$ th user in the $m$ th cell in the $n$th tone is $r_{m k}^{n}=\log _{2}\left(1+\operatorname{SINR}_{m k}^{n}\right)$, and the instantaneous downlink user rate for the $k$ th user over all frequency tones is $R_{m k}=\sum_{n \in \mathcal{D}_{m k}} r_{m k}^{n}$, where the summation is over all the frequency tones assigned to the $k$ th user in the $m$ th cell, i.e. $n \in \mathcal{D}_{m k}$, where $\mathcal{D}_{m k}=\{n \mid k=f(m, n)\}$.

Let the $l$ th base-station send its data to $N_{l}^{n}$ neighboring BSs in the $n$th tone, then, the amount of cooperation required is $N_{l}^{n} r_{l k^{\prime}}^{n}$, where $r_{l k^{\prime}}^{n}$ is the instantaneous data rate of the $k^{\prime}$ th scheduled user in the $l$ th cell in the $n$th tone. Thus, the total cooperation rate of the $l$ th base-station over all frequencies is given by $C_{l}=\sum_{n=1}^{N} N_{l}^{n} r_{l k^{\prime}}^{n}$.

\section{Proposed Algorithm}

\section{A. Problem Formulation}

This paper assumes a network-utility maximization framework. To meet the backhaul-capacity constraints at each BS, while maximizing the network-wide utility, each BS can be thought of as making three decisions at each frequency tone:

1) Cooperation-link selection: Which neighboring BSs should send their user's data stream to this BS so that it can pre-subtract their intercell interference using ZF precoding?

2) User-scheduling: Which user should be served?

3) Precoder-coefficient and power-spectrum adaptation: What are the optimal precoder coefficients to zero-out the intercell interference of the cooperating BSs and what is the optimal power spectra for transmitting data of the scheduled user in its own cell?

These questions must be answered jointly across all the BSs to obtain a network-wide optimal solution. The channels are assumed to vary slowly over time so that the optimization can be done repeatedly as channels vary over time.

The joint cooperation-link selection, user-scheduling, precoder-coefficient and power-spectrum adaptation problem that maximizes the network utility can be expressed as

$$
\begin{array}{ll}
\max & \sum_{m, k} \log \left(\bar{R}_{m k}\right) \\
\text { s.t } & R_{m k}=\sum_{n \in \mathcal{D}_{m k}} \log _{2}\left(1+\mathrm{SINR}_{m k}^{n}\right) \quad \forall m, k \\
& 0 \leq \sum_{l=1}^{L}\left|w_{m k, l k^{\prime}}^{n}\right|^{2} p_{l}^{n} \leq S^{\max } \quad \forall m, n \\
& C_{l}=\sum_{n=1}^{N} N_{l}^{n} r_{l k^{\prime}}^{n} \leq C_{l}^{\max } \quad \forall l
\end{array}
$$

where the optimization is over the cooperation links, the userscheduling function $f(m, n)$, the precoder coefficients $w_{m k, l k^{\prime}}^{n}$ and the power spectra $p_{l}^{n}$. The objective of the problem is to maximize the network-wide utility $\log \left(\bar{R}_{m k}\right)$, which ensures proportional fairness among all users in all cells. Here, $\bar{R}_{m k}$ is the time-averaged rate for the $k$ th user in the $m$ th cell, and $R_{m k}$ is the instantaneous downlink rate of the same user. The second constraint requires that the total transmit power of the $m$ th base-station in the $n$th tone must satisfy the per base-station transmit PSD constraint $S^{\max }$. The third constraint on $C_{l}$ requires that the total cooperation rate of the $l$ th basestation over all frequencies must satisfy the backhaul-capacity constraint $C_{l}^{\max }$.

Dualizing the optimization problem with respect to the backhaul-capacity constraint at each base-station, the optimization problem in (4) can be rewritten as

$$
\begin{array}{ll}
\max & \sum_{m, k} \log \left(\bar{R}_{m k}\right)-\sum_{l=1}^{L} \lambda_{l}\left(\sum_{n=1}^{N} N_{l}^{n} r_{l k^{\prime}}^{n}-C_{l}^{\max }\right) \\
\text { s.t } & R_{m k}=\sum_{n \in \mathcal{D}_{m k}} \log _{2}\left(1+\mathrm{SINR}_{m k}^{n}\right) \quad \forall m, k \\
& 0 \leq \sum_{l=1}^{L}\left|w_{m k, l k^{\prime}}^{n}\right|^{2} p_{l}^{n} \leq S^{\max } \quad \forall m, n
\end{array}
$$

where $\lambda_{l}$ is the Lagrange multiplier which ensures that the backhaul-capacity constraints are met for the $l$ th base-station. $\lambda_{l}$ can also be interpreted as the marginal price of networkutility measured in utility per unit of backhaul capacity of the $l$ th base-station. When $\lambda_{l}$ equals 0 , infinite backhaul capacity is available for the $l$ th base-station and it can cooperate with all the neighboring BSs in all frequency tones to presubtract intercell interference. As the value of $\lambda_{l}$ increases, the backhaul-capacity constraint for the $l$ th base-station decreases to a finite value, until a certain value of $\lambda_{l}$ beyond which there is no cooperation possible as the cost of cooperating with other BSs becomes very high.

The maximization of the marginal increase in the logutility $\log \left(\bar{R}_{m k}\right)$ in (4) can be approximated by maximizing $\left(R_{m k} / \bar{R}_{m k}\right)$. This transforms the objective of the optimization problem in (5), given by $\sum_{m, k}\left(\log \left(\bar{R}_{m k}\right)-\right.$ $\lambda_{m} N_{m}^{n} r_{m k}^{n}$ ), into a weighted rate-sum maximization problem $\sum_{m, k} \alpha_{m k}^{n} r_{m k}^{n}$ with weights for $r_{m k}^{n}$ set as

$$
\alpha_{m k}^{n}=\frac{1}{\bar{R}_{m k}}-\lambda_{m} N_{m}^{n}
$$

\section{B. Overview}

The optimization problem in (4) is a mixed discrete (cooperation-link selection and user-scheduling) and continuous (precoder-coefficient and power-spectrum adaptation) optimization problem. Finding the network-wide optimal solution to (4) jointly across all BSs and tones is impractical. This paper therefore proposes a heuristic approach to solve this optimization problem that first selects the cooperation links for each BS judiciously in each frequency tone (Section III-C). The proposed algorithm then iterates between scheduling the users assuming precoder coefficients and power spectra are fixed, and adapting the precoder coefficients and the power 
spectra assuming the user schedules are fixed. The scheduling of users uses a proportionally fair objective with additional penalty for backhaul-capacity constraint (Section III-D). The ZF precoder coefficients are computed using inversion of the channel limited to cooperating BSs only, and the power spectra are optimized using interior-point method as described in Section III-E.

\section{Cooperation Link Selection}

Adding a cooperation link can reduce the interference to and thus, increase the data rate of the destination BS, but it also incurs a backhaul-capacity cost of sharing the user data over the backhaul link. The first part of the algorithm aims to add the cooperation links which result in the maximum increase in the data rate of the destination BS with a minimum backhaulcapacity cost. However, calculating the benefit of adding each cooperation link is a nontrivial task, because it involves the re-calculation of ZF precoding coefficients and the associated power spectra. In order to devise a practical algorithm for cooperation link selection with the possibility of distributed implementation, this paper proposes to approximate the rate expression with and without cooperation using the following principle. Whenever a cooperation link is added from the $l$ th base-station to the $m$ th base-station in the $n$th tone, it is assumed that the $m$ th base-station would see no interference from the $l$ th base-station in the $n$th tone.

Specifically, let $\left\{\tilde{r}_{m k}^{n}\right\}$ be the initial set of approximate data rates of the scheduled users in the $n$th tone in $L$ cells, given by

$$
\tilde{r}_{m k}^{n}=\log _{2}\left(1+\frac{\left|h_{m, m k}^{n}\right|^{2} p_{m}^{n}}{\Gamma\left(\sum_{j \notin\left\{m, \mathcal{I}_{m}^{n}\right\}}\left|h_{j, m k}^{n}\right|^{2} p_{j}^{n}+\sigma^{2}\right)}\right),
$$

where $\left\{\mathcal{I}_{m}^{n}\right\}$ represents the set of incoming cooperation links at the $m$ th BS in the $n$th tone in the initial topology, and $p_{m}^{n}$ is the transmit power spectrum of the $m$ th BS in the $n$th tone. After adding a cooperation link from the $l$ th base-station to the $m$ th base-station in the $n$th tone (assuming $l \notin \mathcal{I}_{m}^{n}$ ), the new data rate $\tilde{\tilde{r}}_{m k, l}^{n}$ for the $k$ th scheduled user of the $m$ th cell in the $n$th tone is approximated as

$\tilde{\tilde{r}}_{m k, l}^{n}=\log _{2}\left(1+\frac{\left|h_{m, m k}^{n}\right|^{2} p_{m}^{n}}{\Gamma\left(\sum_{j \notin\left\{m, \mathcal{I}_{m}^{n}\right\}}\left|h_{j, m k}^{n}\right|^{2} p_{j}^{n}-\Delta+\sigma^{2}\right)}\right)$,

where the interference from the $l$ th BS to the $m$ th BS, $\Delta=$ $\left|h_{l, m k}^{n}\right|^{2} p_{l}^{n}$, is assumed to be absent. Clearly, this is only an approximation as the exact rate expression is a complicated function of the ZF precoding coefficients and the optimized power spectra. Nevertheless, the approximation is a fairly good one, especially when the channel is diagonally dominant.

The approximated rate expressions (7) and (8) are now used for cooperation link selection. As the objective is to maximize the network utility $\sum_{m, k}\left(\bar{R}_{m k} / R_{m k}\right)$, the benefit of adding a cooperation link from the $l$ th base-station to the $m$ th basestation in the $n$th tone is approximately given by $\left(\tilde{\tilde{r}}_{m k, l}^{n}-\right.$ $\left.\tilde{r}_{m k}^{n}\right) / \bar{R}_{m k}$.
The backhaul-capacity cost of adding the cooperation link from the $l$ th base-station to the $m$ th base-station in the $n$th tone approximately equals the cost of transmitting user data of the source base-station $l$ over the backhaul given by $\lambda_{l} \tilde{r}_{l k^{\prime}}^{n}$. Note that the cost of transmitting additional user data corresponding to the increased data rate of the destination BS over the outgoing links from the destination BS is ignored here. Thus, the algorithm selects the cooperation link from the lth basestation to the $m$ th base-station in the $n$th tone if

$$
\frac{1}{\bar{R}_{m k}}\left(\tilde{r}_{m k, l}^{n}-\tilde{r}_{m k}^{n}\right)>\lambda_{l} \tilde{r}_{l k^{\prime}}^{n},
$$

accounting for the benefit and the cost of adding this link to the initial topology. This estimation can be done at the $m$ th BS and then communicated to the $l$ th BS.

\section{Proportionally Fair Scheduling with Backhaul-Capacity Penalty}

The approximate rate expression (7) can also be used to schedule the active user in each cell and in each frequency tone. Assuming that the cooperation links and the power spectra are fixed, a proportionally fair scheduler can select the active user while accounting for an additional penalty for the backhaul-capacity constraint. The user scheduling step assigns user $k$ in each frequency tone $n$ at the $m$ th $\mathrm{BS}$ as

$$
f(m, n)=\operatorname{argmax}_{k}\left(\frac{1}{\bar{R}_{m k}}-\lambda_{m} N_{m}^{n}\right) \tilde{r}_{m k}^{n},
$$

where $\tilde{r}_{m k}^{n}$ is the instantaneous approximate data rate of the $k$ th user in the $m$ th cell in the $n$th tone $n$ computed using (7). The above scheduling policy maximizes the log-utility, but also includes additional backhaul-capacity cost of $\lambda_{m} N_{m}^{n} \tilde{r}_{m k}^{n}$ of transmitting user data over the $N_{m}^{n}$ outgoing cooperation links from the $m$ th BS.

\section{E. Zero-Forcing Precoder Coefficient and Power Adaptation}

With the cooperation links and the user schedule fixed, we can now compute the ZF precoder and the optimal power spectra. The ZF precoding coefficients are chosen such that if there is an outgoing cooperation link from the $l$ th base-station to the $m$ th base-station, then the scheduled user of the $m$ th base-station sees no intercell interference from the $l$ th cell. The power spectra of the BSs are optimized to ensure that the per-BS power constraints are satisfied. This is a nontrivial step, which needs to be done using nonlinear optimization methods for the maximization of the objective in (4). In the remainder of this section, as user scheduling is fixed, the user subscript $k$ is dropped for notational convenience.

1) Channel Inversion: For the full-cooperation scenario, the precoding matrix can be computed as the inverse of the channel gain matrix. For the scenario where each BS cooperates with only a subset of the neighboring BSs, it is shown below that the precoding coefficients can be computed by inverting the channel gain matrix restricted to the cooperating BSs only.

For all the outgoing cooperation links from the $m$ th basestation to the $l$ th base-station in the $n$th tone, the following condition holds

$$
\left(\mathbf{h}_{l}^{n}\right)^{H} \mathbf{w}_{m}^{n}=0 .
$$


This condition means that the $m$ th base-station sends its user's data to the $l$ th base-station via backhaul link and the $l$ th basestation uses zero-forcing precoding coefficients $w_{l, m}^{n}$ to zeroout the intercell interference from the $m$ th cell to the scheduled user in its own cell. Let the $m$ th base-station send its user's data in the $n$th tone to $N_{m}^{n}$ neighboring BSs, and let the indices of these BSs be represented by the set $\mathcal{O}_{m}^{n}$. Define the channel gain matrix as $\mathbf{H}^{n}=\left[\mathbf{h}_{1}^{n}, \mathbf{h}_{2}^{n}, \cdots, \mathbf{h}_{L}^{n}\right]^{H}$, where $(.)^{H}$ represents the hermitian operator. Then, (11) can be rewritten as

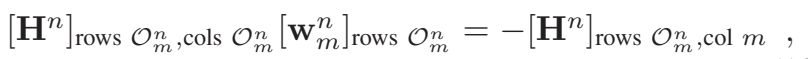

where $[T]_{\text {rows }} \mathcal{K}_{1}$, cols $\mathcal{K}_{2}$ represents the matrix defined by choosing only the rows in set $\mathcal{K}_{1}$ and the columns in set $\mathcal{K}_{2}$ from

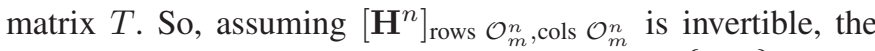
non-zero zero-forcing precoding coefficients in $\left\{\mathbf{w}_{m}^{n}\right\}$ can be computed by

$$
\left[\mathbf{w}_{m}^{n}\right]_{\text {rows } \mathcal{O}_{m}^{n}}=-\left(\left[\mathbf{H}^{n}\right]_{\text {rows } \mathcal{O}_{m}^{n}, \text { cols } \mathcal{O}_{m}^{n}}\right)^{-1}\left[\mathbf{H}^{n}\right]_{\text {rows } \mathcal{O}_{m}^{n}, \text { col } m} \cdot
$$

Note that $w_{l, m}^{n}=0 \forall l \notin\left\{\mathcal{O}_{m}^{n}, m\right\}$ and $w_{m, m}^{n}=1 \forall m$.

2) Power Spectrum Adaptation: The power-spectrum adaptation step assumes that the precoder coefficients are computed using (13), and proceeds to optimize the transmit power spectra by solving the network-utility maximization problem (5). The maximization of the marginal increase in log-utility while accounting for the penalty in backhaul gives a weighted rate-sum maximization problem on a tone-by-tone basis with weights for $r_{m k}^{n}$ set as $\alpha_{m k}^{n}=\frac{1}{\bar{R}_{m k}}-\lambda_{m} N_{m}^{n}$, i.e.

$$
\begin{array}{ll}
\max & \sum_{m, k} \alpha_{m k}^{n} r_{m k}^{n} \\
\text { s.t } & 0 \leq \sum_{l=1}^{L}\left|w_{m k, l k^{\prime}}^{n}\right|^{2} p_{l}^{n} \leq S^{\max } \quad \forall m
\end{array}
$$

where the optimization is over the transmit power spectra $\left\{p_{m}^{n}\right\}$. Here, the inequality constraint on power spectra requires that the total transmit power spectra of the $m$ th $\mathrm{BS}$ in the $n$th tone must satisfy the transmit PSD constraint $S^{\text {max }}$. Define the equivalent channel matrix $\tilde{H}^{n}$ as containing the channel gains $\tilde{h}_{l, m}^{n}=\left(\mathbf{h}_{m}^{n}\right)^{H} \mathbf{w}_{l}^{n}$, then, the instantaneous data rate in the $n$th tone can be rewritten as

$$
r_{m k}^{n}=\log _{2}\left(1+\frac{\left|\tilde{h}_{m, m}^{n}\right|^{2} p_{m}^{n}}{\Gamma\left(\sum_{l \neq m, l=1}^{L}\left|\tilde{h}_{l, m}^{n}\right|^{2} p_{l}^{n}+\sigma^{2}\right)}\right) .
$$

The optimization problem in (14) is non-convex in $\left\{p_{m}^{n}\right\}$. This paper adopts an interior-point method [12] to find a local optimum of (14). In particular, in the Newton-step computation for interior-point method, the off-diagonal terms of the Hessian are ignored and the Newton step is computed as the ratio of the gradient and the absolute-value of the diagonal of the hessian (because the objective is not concave) to get an increment direction in each step [13]. Note that power-spectrum adaptation is a crucial step of the network optimization problem (4). This is because the power needed for zero-forcing the interference is non-negligible, and an alternative approach of treating the inequality constraint as an equality to compute $\left\{p_{m}^{n}\right\}$ directly can result in negative powers, which are infeasible.

\section{F. Summary}

The overall algorithm is summarized below:

1) Initialize $p_{m}^{n}=S^{\max } \forall m, n$. Use joint scheduling and dynamic power-spectrum adaptation algorithm [13] to get an initial set of data rates $\left\{r_{m k}^{n}\right\}$ and power spectra $\left\{p_{m}^{n}\right\}$ at each base-station.

2) Select cooperation links using (9) with the power spectra $\left\{p_{m}^{n}\right\}$ from Step 1). Here, a set of $\left\{\lambda_{m}\right\}$ are chosen for all BSs based on the backhaul-capacity constraints.

3) Fix the cooperation links and repeat:

a) Schedule the users using (10) with the power spectra $\left\{p_{m}^{n}\right\}$ from last iteration.

b) Fix user schedules and compute the ZF precoder coefficients using (13). Then, optimize the power spectra $\left\{p_{m}^{n}\right\}$ by solving (14) using interior-point method .

c) Update the proportional-fairness weights $\left\{\alpha_{m k}^{n}\right\}$.

4) Update Lagrange multipliers $\left\{\lambda_{m}\right\}$ and return to Step 2) if backhaul-capacity constraints are not met. Lagrange multipliers can be updated using subgradient method, i.e. $\lambda_{m}=\left[\lambda_{m}+s\left(C_{m}-C_{m}^{\max }\right)\right]_{+}$, where $s$ is the step-size and $[a]_{+}=\max (0, a)$.

Step 3 is repeated for a fixed number of iterations. In addition, we keep only the values which increase the objective in (5) to ensure the convergence of the algorithm.

\section{Simulation Results}

The performance of the proposed algorithm is evaluated on a wireless cellular network with 7 cells and 40 users per cell, with maximal frequency reuse, where cells are wrapped around so that each cell has six neighboring cells. Standard cellular network parameters are used in simulation: the noise power spectral density is set to $-162 \mathrm{dBm} / \mathrm{Hz}$; frequencyselective channel vectors over 64 frequency tones are chosen according to the multipath time delay profile of ITU-R M.1225 PedA and a distance-dependent path-loss model $L=128.1+$ $37.1 \log _{10}(d)$, where $d$ is the distance in $\mathrm{km}$, with $8 \mathrm{~dB} \log$ normal shadowing and a Rayleigh component. Perfect channel estimation is assumed for the downlink channel. The distance between neighboring BSs is set to $1.4 \mathrm{~km}$. An antenna gain of $15 \mathrm{dBi}$ is assumed. The maximum PSD constraint is -27 $\mathrm{dBm} / \mathrm{Hz}$, so that over a $10 \mathrm{MHz}$ bandwidth the total transmit power at the BS is $49 \mathrm{dBm}$. The initial user-assignment is random. For evaluation purposes, the channels are assumed to be fixed throughout and $\lambda_{m}$ is assumed to be equal for all cells, which corresponds to a backhaul-capacity constraint on the sum of backhaul capacities of all BSs.

Fig. 2 shows the improvement in downlink sum-rates per cell as a function of the backhaul capacity per BS. The most notable feature is that achievable downlink sum-rate scales linearly as the average backhaul capacity per BS increases from the no-cooperation scenario to the full-cooperation scenario. The no-cooperation scenario uses joint proportionally 


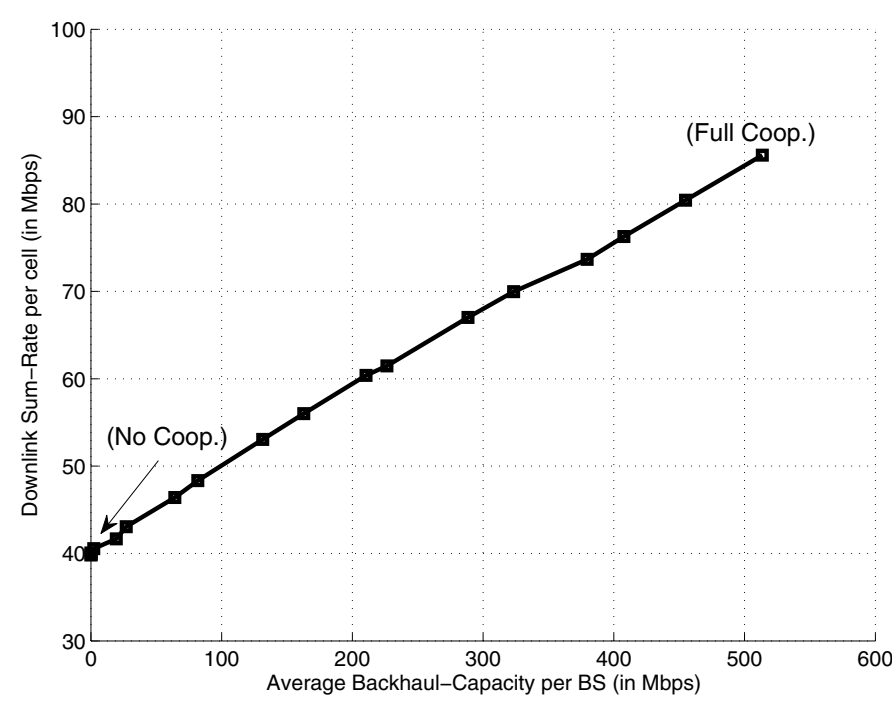

Figure 2. Downlink sum-rate per cell vs backhaul capacity per BS.

fair scheduling and dynamic power-spectrum adaptation [13]. In the full-cooperation scenario, each BS uses ZF precoding to zero-out the interference of all the neighboring BSs on all tones and thus, each BS shares its users' data streams with all the neighboring BSs on all tones. Thus, the required backhaul capacity is six times the average downlink sum-rate of each cell. However, if, for example, 50\% of the full-cooperation backhaul capacity is available, then the gain in downlink sumrate as compared to the no-cooperation scenario becomes $50 \%$. Thus, the proposed algorithm provides a network operator a range of possibilities to balance between the benefit and the cost of using backhaul capacities for intercell interference mitigation.

Fig. 3 shows the cumulative distribution functions of the downlink user rates for different backhaul capacities (per BS). It can be seen that the gains of ZF precoding are distributed fairly among the different users, i.e., both the 10th and the 90th percentile users obtain almost equal percentage gain from the presence of cooperation links in the network.

\section{CONCLUSiOns}

This paper proposes a numerical algorithm to investigate the gains from multicell cooperative processing in a wireless multicell OFDMA downlink network when the backhaul capacity of each BS is limited. The proposed algorithm maximizes the network-wide utility subject to backhaul-capacity constraints and with a proportional fairness objective to ensure a fair distribution of gains among the users. This algorithm selects a subset of tones at each BS for cooperation with the neighboring BSs to utilize the limited backhaul capacity judiciously and then, iteratively updates the user schedule, the precoder coefficients and the power spectra. Numerical results suggest a linear trade-off between the gain in downlink sum-rate per cell and the average backhaul capacity per BS as the backhaulcapacity per BS increases from the no-cooperation scenario to the full-cooperation scenario. This illustrates that the benefit of multicell cooperative processing can be obtained gradually as backhaul capabilities improve in a wireless multicell network.

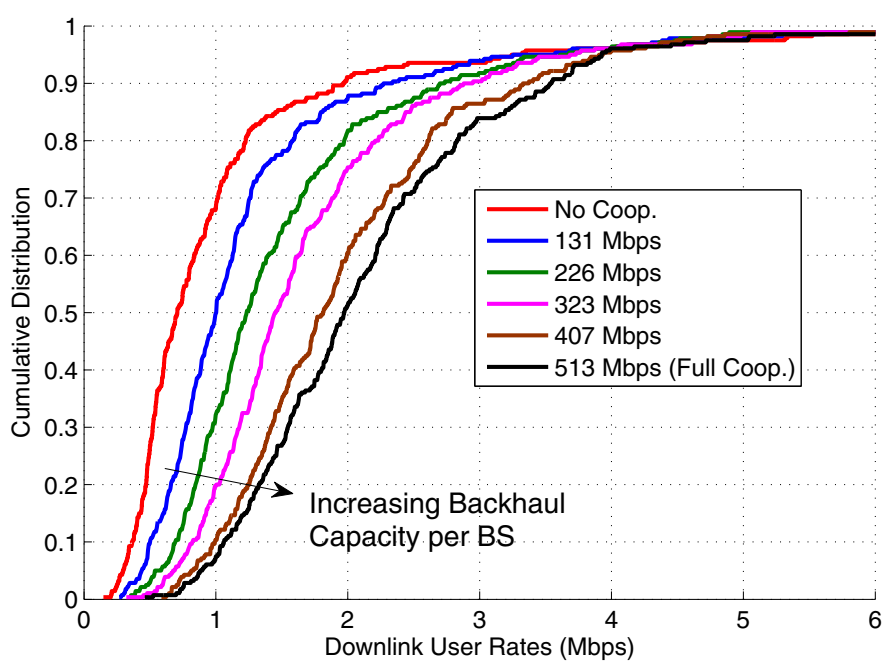

Figure 3. Cumulative distribution functions of downlink user rates for different backhaul capacities per BS.

\section{ACKNOWLEDGEMENTS}

The authors wish to thank Mr. Anubhav Singla for performing an initial set of simulations for the problem defined in this paper in a MITACS internship program at the University of Toronto, Canada.

\section{REFERENCES}

[1] S. Jing, D. N. C. Tse, J. B. Soriaga, J. Hou, J. Smee, and R. Padovani, "Multicell downlink capacity with coordinated processing," EURASIP J. Wirel. Commun. Netw., vol. 2008, pp. 1-19, 2008.

[2] H. Zhang and H. Dai, "Cochannel interference mitigation and cooperative processing in downlink multicell multiuser MIMO networks," EURASIP J. Wirel. Commun. Netw., vol. 2004, no. 2, pp. 222-235, 2004.

[3] D. Gesbert, S. Hanly, H. Huang, S. Shamai, O. Simeone, and W. Yu, "Multicell MIMO cooperative networks: A new look at interference," IEEE J. Select. Areas Commun., vol. 28, no. 9, pp. 1380-1408, Dec. 2010.

[4] H. Huang and M. Trivellato, "Performance of multiuser MIMO and network coordination in downlink cellular networks," in 6th Int. Symp. on Modeling and Optimization in Mobile, Ad Hoc, and Wireless Networks (WiOPT), Apr. 2008, pp. 85-90.

[5] A. Papadogiannis, D. Gesbert, and E. Hardouin, "A dynamic clustering approach in wireless networks with multi-cell cooperative processing," in Proc. IEEE Inter. Conf. Commun. (ICC), May 2008, pp. 4033-4037.

[6] J. Zhang, R. Chen, J. Andrews, A. Ghosh, and R. Heath, "Networked mimo with clustered linear precoding," IEEE Trans. Wireless Commun., vol. 8, no. 4, pp. 1910-1921, Apr. 2009.

[7] S. Venkatesan, "Coordinating base stations for greater uplink spectral efficiency in a cellular network," IEEE 18th Int. Symp. Personal, Indoor and Mobile Radio Commun. (PIMRC), pp. 1-5, Sep. 2007.

[8] F. Boccardi and H. Huang, "Limited downlink network coordination in cellular networks," in IEEE 18th Int. Symp. Personal, Indoor and Mobile Radio Commun. (PIMRC), Sep. 2007, pp. 1-5.

[9] P. Marsch and G. Fettweis, "On multi-cell cooperative signal processing in backhaul-constrained cellular systems," Annals of Telecommunications, vol. 63, no. 5, pp. 253-269, 2008.

[10] S. Hari and W. Yu, "Partial zero-forcing precoding for the interference channel with partially cooperating transmitters," in Proc. IEEE Inter. Symposium on Inform. Theory (ISIT), Jun. 2010, pp. 2283-2287.

[11] A. Papadogiannis, H. Bang, D. Gesbert, and E. Hardouin, "Downlink overhead reduction for multi-cell cooperative processing enabled wireless networks," in IEEE 19th Int. Symp. Personal, Indoor and Mobile Radio Commun. (PIMRC), Sep. 2008, pp. 1-5.

[12] S. Boyd and L. Vandenberghe, Convex Optimization. Cambridge, 2004.

[13] W. Yu, T. Kwon, and C. Shin, "Joint scheduling and dynamic power spectrum optimization for wireless multicell networks," in 44th Annual Conf. on Information Science and Systems (CISS), Mar. 2010, pp. 1-5. 